SYMPOSIUM ON EVIDENCE BASED MEDICINE

\title{
Evidence based medicine guidelines: a solution to rationing or politics disguised as science?
}

\author{
S I Saarni, H A Gylling
}

See end of article for

authors' affiliations

J Med Ethics 2004;30:171-175. doi: 10.1136/jme.2003.003145

Correspondence to:

S Saarni, National Public

Health Institute,

Department of Mental

Health and Alcohol

Research,

Mannerheimintie 166 00300 Helsinki, Finland;

samuli.saarni@helsinki.fi

Revised version received 19 June 2003

Accepted for publication

7 August 2003

\begin{abstract}
"Evidence based medicine" (EBM) is often seen as a scientific tool for quality improvement, even though its application requires the combination of scientific facts with value judgments and the costing of different treatments. How this is done depends on whether we approach the problem from the perspective of individual patients, doctors, or public health administrators. Evidence based medicine exerts a fundamental influence on certain key aspects of medical professionalism. Since, when clinical practice guidelines are created, costs affect the content of EBM, EBM inevitably becomes a form of rationing and adopts a public health point of view. This challenges traditional professionalism in much the same way as managed care has done in the US. Here we chart some of these major philosophical issues and show why simple solutions cannot be found. The profession needs to pay more attention to different uses of EBM in order to preserve the good aspects of professionalism.
\end{abstract}

$\mathrm{T}$ he costs of health care have been escalating for decades. Lately, the speed of this development has become harder to accept as it has greatly outpaced the growth of the national economy in many countries. The multiple reasons for this trend include the growth in expensive technologies and increasing demand for services fuelled by a complex mix of social changes-for example, improved levels of education, the commodification of medicine, and the general medicalisation of life.

There is also rising demand for quality improvement and quality control in health care. Some of the equally complex social developments underlying this are the increasing autonomy, rights, and knowledge of patients; commodification of the doctor patient relationship towards a provider client relationship; declining trust in professionalism and professional judgment; greater trust in statistical research; and less toleration of medical error. These trends affect the different parties involved in health care-patients, doctors, and administrators or payers-differently, and can lead to conflicts of interest.

It has also become commonplace to argue that increasing resources will not necessarily produce any good if not spent effectively. ${ }^{1}$ Thus, when more money is promised for health care, it is done on the condition that it can be proved that the money is spent on effective interventions. This trend has created an unprecedented need for the medical profession to explicitly justify its actions in both medical and economic terms. Evidence based medicine (EBM), or the whole outcomes movement, ${ }^{2}$ is a central tool in this process of increasing the accountability of medicine.

Cost control and quality improvements are often combined in practical health care development - to get better for less is an obvious ideal for health care administrators. Even if, however, this might be logical and practical on the administrative level, for individual patients and doctors it is often the cause of much of the confusion that surrounds both EBM and rationing. We argue that this confusion of costs and quality has made it difficult for doctors to foresee and react to the changes EBM implies for the whole profession.

\section{EVIDENCE BASED MEDICINE}

The definition of evidence based medicine is ambiguous, and the term acquires different meanings in different contexts. This leads to confusion, because the explicit definitions provided do not match all the uses of the term in practice. Commonly, EBM is defined in a generally positive and individualistic way that emphasises the importance of outcomes and states, more or less, that a doctor makes his/her decisions according to the best available knowledge, and that this knowledge is acquired by the best possible empirical scientific methods. ${ }^{23}$ Evidence based medicine represents an empiricist mode of thinking in medicine. ${ }^{4}$ Definitions of this type have undoubtedly been of great practical significance in improving medical care and research. They complicate the discussion about the outcomes movement, however, by being vague in factual terms but clear in moral meaning. A critique of EBM - for example - can be interpreted as being against the best interests of patients and thus against traditional medical ethics. As EBM has become a politically influential doctrine, however, a rational discussion of all sides of EBM is long overdue for the medical profession. Attempts to cover real dilemmas with sarcasm ${ }^{5}$ might not facilitate a balanced and useful debate.

\section{Definitions: epistemological and practical EBM}

It appears helpful to separate two partly overlapping uses of EBM: ${ }^{2}$ first, as an epistemological term setting the hierarchy and the gold standard of medical knowledge (epistemological EBM), and second, as a term describing the optimal way to practise medicine (practical EBM).

The central focus of epistemological EBM is to emphasise the probabilistic knowledge acquired via clinical trials, ideally double blind randomised controlled trials (RCTs), over other forms of knowledge-for example, intuition, clinical authority, and pathophysiological theories. The main tool of epistemological EBM is systematic review of available scientific evidence. Systematic reviews are-or in theory can be simplified as-factual statements: A (condition) + B (treatment) $=\mathrm{X}$ (outcome) with probability of $\mathrm{p}$. 
Practical EBM, on the other hand, describes a certain optimal way to practise medicine. The central tools of practical EBM are clinical practice guidelines (CPGs) that are-or, again, can be simplified as—recommendations: if A, do B.

In practical EBM the epistemological and practical components are combined: if $\mathrm{A}$, do B, because $\mathrm{X}$ will follow $\mathrm{B}$ with probability p.

Epistemological and practical EBM are commonly confused, and both are simply called "EBM". This confusion has serious consequences. We emphasise that EBM matters only when applied to practice, and thus the debates around epistemological EBM are only relevant in connection with practical EBM. Thus, by CPGs we always mean EBM CPGs, that is guidelines that are related in some way to the principles of evidence based medicine. (In theory, guidelines could, of course, also be totally arbitrary, or politically or culturally dictated.)

\section{Practical challenges of EBM}

There are several practical challenges facing EBM, and many approaches to solving them. Some of the challenges are:

- possible quality problems in finding and summarising available evidence

- lack of relevant and clear evidence in many areas of medicine

- the fact that research populations are often more homogeneous than actual patient populations

- the impossibility of conducting double blind RCTs in all fields of medicine

- the impossibility of including in scientific research all possible clinical details that might be relevant when treating individual patients

- the importance of non-medical knowledge in creating guidelines-for example, patient, practitioner, and organisational preferences, or cultural, social, political, and economic factors-that may render otherwise commendable guidelines inapplicable. ${ }^{6}$

Despite attempts to diminish the practical problems of EBM, some theoretical problems remain that cannot be resolved by empirical research. These theoretical problems affect the solutions to the practical challenges listed above, although to differing extents. We consider four priority issues that arise when moving from scientific results to CPGs: the nature of evidence; the interpretation of evidence; the consideration of costs; and the application of CPGs in practice. We hope our relative lack of discussion of some of the uncontroversially positive aspects of EBM is excused, as we feel they are generally better covered in the literature.

\section{What is evidence in EBM}

An important question about the definition of EBM is whether there is an evidence based medicine solution to all medical problems. If the answer is yes, EBM means the currently best available knowledge-even if this might be "only" professional intuition. ${ }^{3}$ If the answer is no, EBM denotes a certain level of quality of knowledge-for example, a specified methodological quality of scientific studies, or a small enough $p$ value of their results. If this quality is exceeded, the knowledge can be called evidence. Both interpretations of the term, but especially the former, also explain why EBM is a positively evaluative concept with powerfully attractive properties. The former interpretation, although more true to the ideas of the founders of the EBM movement, risks the meaningful use of the term EBM, as it easily becomes synonymous with good medical practice which should be the obvious ethical norm. We think following evidence has always been important to good medical practice - at least in theory, if not always in practice.

The literature around EBM has an abundance of clear hierarchies and methods for grading the quality of evidence, but it deals less with the question of evidence versus nonevidence. This is understandable for several reasons. First, this problem cannot be thoroughly resolved using the methods of empirical science. Second, for the individual patient or doctor it might appear irrelevant. Medicine on an individual level is pragmatic, and what matters is that patients are treated in the best possible way-that is, according to the best available knowledge. Third, since the methodological ideals of EBM are statistical and inductive, we can never have totally failsafe knowledge even if we know the results of relevant high quality RCTs. The best we can have are increasing probabilities of certain outcomes.

In practice, however, the division between evidence and non-evidence must always be drawn-and this should be done explicitly-because it has important practical and political implications. First, clinicians rarely base their actions on raw scientific data, but for practical reasons rely on textbooks, reviews, meta-analyses, or CPGs, where the question of the evidence threshold has usually been resolved. Second, knowledge about a subject is sometimes so poor that the doctor might opt for doing nothing instead of basing his or her actions on guesswork (emphasising the maxim "first, do no harm" over the possibility of benefit.). ${ }^{7}$ Third, public health administrators increasingly claim to rely on EBM when searching for evidence of the cost effectiveness of a treatment. If there is no such evidence, it should not be financed, and professional intuition is rarely regarded as evidence in these debates. The same is true of individual patients' own experiences, opinions, and preferences.

This link between economics and health policy complicates the definition of evidence. For most medical practice a hierarchy of evidence would be sufficient, but this is not so for public health policy. It can be argued that limiting the use of EBM to situations where there is evidence of a certain quality would give the term a clearer meaning by differentiating it from vague "good medical practice". We argue that this shift of meaning has already happened, and that it will have important practical and ethical consequences. One simple reason for this is that some fields of medicine are more difficult to research than others, due to methodological or economical limitations.

\section{The interpretation of evidence}

The definition of evidence is important, as discussed above, because it illustrates the struggle between patients, scientists, doctors, and public health administrators over the interpretation of scientific results and how to decide the proper goals of medicine. This conflict marks what we might think of as the second, political phase of EBM, which differs from the first phase where EBM is advocated as a purely medical endeavour for the equal good of all. In some respects this is a comparable and related development to that surrounding the rationing of health care, -for example, Holm (1998) ${ }^{8}$. Political should encompass ethical values, but it is possible that ethical considerations are subordinated to political and economic aims.

Evidence can be interpreted in many ways, partly because the knowledge we acquire through clinical trials, especially the RCT, is statistical in nature (meaning that the results are based on effects observed on populations). In combination with the selection of study variables, outcome units, and level of statistical significance, this inevitably leads to uncertainty in dealing with individual patients. Despite this rather obvious shortcoming, however, it is also true that the desire for certainty in medicine and the minimisation of clinical 
variance (differences in treatments between patients, doctors, and areas) have been important driving forces of the EBM movement from the beginning. ${ }^{4}$

\section{Statistical uncertainty, individuals, and public health}

The explanation for this anomalous state of affairs is that the uncertainty created by statistical results is less an issue for public health administrators than for individual patients and doctors. To say that results of clinical studies are statistical is, of course, a simplification, because different types of statistical results can be very different in their theoretical and clinical implications. ${ }^{10}$ We consider two central examples to illustrate the point.

First, it is common that we know with a great certainty (small $\mathrm{p}$ value) that a treatment is effective for a certain percentage of patients. In other words, when a certain number of patients ("number needed to treat", NNT) is treated, one patient will benefit. For the individual patient and physician the situation is uncertain: there is no way of knowing whether the patient will benefit. For the public health administrator, however, there is little uncertainty: if there are enough cases, the consequences of the treatment on the population can be estimated and resource implications calculated.

Second, it is common with new, experimental treatments that we have positive but uncertain results from clinical studies. This means that results are promising but it is possible that they are created by chance-that is, the $p$ value is large. Here the uncertainty is greater than in the previous example: it still concerns the individual, but now also the scientists and public health administrators. Nobody knows for certain if the treatment will work.

\section{Different stakeholders and rationales}

How should these results be interpreted? In both cases, if the disease is serious enough and the treatment is not associated with risks or costs, the patient is most certainly rational to want treatment. ${ }^{11}$ However, how should the distributor of public resources interpret the evidence? This is a central question for evidence based priority setting.

The first example shows how different groups have a rational interest in balancing the evidence differently. Even if public health is obviously always related to real individuals' health, the sum of public health is produced by many different individual distributions of health. So even if we believe that maximising public health-however definedshould be the overriding aim of the health care system, we still need considerations of justice. In the second example the disagreement is on a different level. Patients with a serious disease have an interest in not rejecting a true hypothesis (type 2 statistical error) for an obvious reason: they might die before the hypothesis is sufficiently validated. Scientists, on the other hand, generally try to avoid accepting a false hypothesis (type 1 statistical error). ${ }^{12}$ Public health administrators might not finance treatments without proven effectiveness. So, in both cases, when rationing is needed the issue of interpreting evidence becomes political, and separates the interests of patients from those using EBM for rationing purposes..$^{13} 14$

What, however, should the doctor do? According to traditional medical ethics he/she should recommend treatment in both cases, but according to EBM this is not certain, especially in the second case. The recommendation depends on how the political component of practical EBM is managed and what the opportunity costs are. This exemplifies how practical EBM changes the way we think about the role of the doctor, and how it is slowly moving the emphasis of medical ethics from individuals to public health, and from benefiting patients to avoiding harm.

\section{Cost considerations}

The first question about costs in EBM is whether they are to be considered at all. Epistemological EBM does not require cost considerations, but practical EBM does. Costs inevitably play a role when CPGs are created and individual patients treated, even if in many cases only implicitly, for at least two reasons. First, because for CPGs to be relevant their recommendations must be realistic - that is, possible to apply in practice. A guideline recommending a treatment that nobody can or wants to provide is futile, and more likely to be damaging if there is no realistic alternative recommendation-for example, Western HIV treatment guidelines in most African countries. Second, because opportunity costs-that is, the alternative goods (not just monetary) that will be lost if a treatment is given-must be considered. Considering costs as part of trying to find the best possible solution to an individual patient's problem is a traditional and important part of medical praxis. It is common that patients do not buy medication they consider too expensive, or use a lower dosage than prescribed, without reporting this to the doctor. Patients always consider opportunity costs on their individual level, and these naturally depend on the way the health care is financed. On the individual level, cost considerations can in theory be dealt with relatively simply by letting individual patients decide what they are willing to pay for certain services. In insurance based systems people can choose the insurance policy they like and can afford, although, at least in the US, this appears true in most cases only in theory..$^{15}$ And even in theory, it is quite optimistic to think we could correctly predict our reactions to some possible future diseases and ailments that we have no experience of at the moment.

How opportunity costs are considered when creating CPGs depends largely on who is writing the guidelines-for example, a national governmental body; a group of medical specialists; a patient organisation; or a managed care organisation (MCO). Different groups have different opportunity costs, because they may only concentrate on a certain group of patients or diseases, and may ignore the benefits provided for other groups. This is less possible when approaching the question from a public health point of view-for example, as a governmental organisation in a country with a public health care system. There is still, however, the problem of comparing the different aims and goals of health care (cure, care, security, justice, etc.) with each other, and also of valuing the many health care related goods against other possible societal goods. These are political decisions and ethical questions. This shows-again-how the problems of rationing are central to practical EBM as well.

The consideration of opportunity costs places the doctor in a difficult position. Whose interests should he/she primarily consider? More concretely, how should the different opportunity costs and interests of payers and patients be balanced? On the one hand there is the traditional, atomistic, medical ethics that most doctors value and that arguably is important for the trust the public has in the profession. On the other hand, there is the fact that modern medicine may be poorly practised without some third party financing the services. ${ }^{16}$ Can the doctor stand on both sides, and should he/she even try, as suggested by some prominent professionals? ${ }^{17}{ }^{18}$ Well aware of traditional medical ethics, many MCOs have created economic incentives to attract doctors to their side, as have wealthy patients. ${ }^{19}$ Publicly funded health care systems will probably follow, but possibly using different methods in different countries. ${ }^{20}$ 
Perhaps paradoxically, many appear to base their solution to this dilemma on science and EBM: it is hoped that scientific objectivity will provide clear answers which no personal, moral, or economic loyalties can confound. As we have tried to show, EBM cannot solve these problems nor make them disappear.

\section{Practical EBM is a form of rationing and should be considered as such}

If cost considerations are inevitable in creating evidence based CPGs, and if they lead to some potentially beneficial treatments not being recommended, this makes practical EBM a form of rationing. ${ }^{21}{ }^{22}$ This has important implications for the legitimisation of CPGs. A strong argument can be made that the problems of rationing cannot be solved by rational, simple rules, but instead require a fair and politically transparent process of decision making. ${ }^{82} 24$ The implication is that if rationing decisions are inherently political, creating CPGs becomes political too, and CPGs created by purely professional groups are as legitimate as the rationing decisions made by these groups. Many systems for creating guidelines note that value judgments must be made somewhere in the process of guideline creation, but fail to state clearly how these judgments should be made. ${ }^{25-27}$ Often, vague procedural advice recommending a multiprofessional approach is given. To be more concrete, however, we should see how rationing decisions can be made. We could-for example-apply the procedural model for rationing developed by Daniels and Sabin ${ }^{24}$ to guideline creation. "Evidence based rationing" cannot be a simple and linear application of scientific results to health policy. ${ }^{28} 29$

\section{Applying CPGs}

A common complaint of guideline creators is that practising physicians do not follow their guidelines in large enough numbers. This complaint is understandable when we remember that reducing clinical variation and uncertainty is a central goal of EBM, which is in turn believed to lead to better medical outcomes. This attitude also shows how practical EBM is more a political movement aimed at influencing practice, than a scientific endeavour. The crucially important aspect of practical EBM is how it changes the practice of doctors. Central to this is how CPGs are implemented and followed: first, are they seen as the minimum or maximum of care; second, do they bind clinicians or payers in any way?

In practice, these questions are obviously related to the health care system and to who has created the guidelines. Different guidelines will be applied very differently. In theory there are three main options. First, CPGs can, and usually are, seen as recommendations or guidelines which clinicians apply to individual situations in the way they see best. Second, guidelines can stipulate the maximum of care, by setting limits to the care being financed. Third, guidelines can set the minimum of care by defining the legal norm (below which the treatment can be seen as malpractice) and the minimum of care that must be financed.

Since varying applications of different guidelines could create unnecessary confusion, it is important to have a clear understanding of how different stakeholders relate to guidelines. ${ }^{30}{ }^{31}$ It is possible, however, that the same guideline could be used for all these three purposes in different contexts. This could even be likely if we consider the global trends mentioned at the beginning-increasing need for cost control, quality improvement, and legal protection for patients and doctors. Clinical practice guidelines might fulfil these goals better if they were tightly adhered to. ${ }^{1}$ This suggests that guidelines-at least if they are produced with any form of contact with the financiers of the health care, as they should be to be truly applicable-will become more and more binding in the long run.

The possibility then arises that guidelines become binding recommendations which bind clinicians by stating the minimum and the maximum of possible care to be the same. This would lead to an inevitable decline in practical professional autonomy, even if no EBM enthusiast we know of wants to advocate "cookbook medicine" or talk about binding guidelines. It is important to note that limitations of professional autonomy will in practice also limit the autonomy of patients to choose their treatments.

Throughout this article, we have only considered rational reasons that different stakeholders might have for different treatment choices, but limitations of autonomy can also be supported by claims of irrationality. Evidence based medicine can-for example-be seen as a solution to the problem of drug company influence over medicine: limitations on professional and patient autonomy are justifiable, because both groups make irrational decisions based on commercial marketing. ${ }^{32}$

\section{CONCLUSIONS: EBM, RATIONING, AND TRADITIONAL MEDICAL ETHICS}

We have discussed some conceptual and practical problems that arise when EBM is applied to practice in medicine and health care. A common factor in these problems seems to be that EBM is so ambiguous a concept that it can be used differently in different contexts. Even if the original definitions of EBM emphasised the individual level, there are many reasons why emphasis has shifted more and more to the level of public health. We have tried to show that even if one wants to use EBM purely as a scientific tool aimed at quality improvement at the individual level, this is not entirely possible. The problems of rationing are relevant to the discussion about EBM, and if rationing is to be seen as an openly political process that must not be disguised as science, the same should apply to EBM as well.

Evidence based medicine has importance for the conflict between traditional Hippocratic medical ethics and modern medicine. Traditionally, the doctor has fulfilled his professional duty by striving to do the best for the individual patient in all circumstances, without considering the opportunity costs of his actions. This atomistic approach has emphasised the uniqueness of the individual patient and the importance of the doctor patient relationship. Modern medicine can rarely be atomistic, however, as it is dependent on multiprofessional teams, expensive technology, and some sort of collective payer. This means that the payers have an increasingly powerful role in health care decision making. They enforce EBM from the public health point of view, and require that doctors and patients consider resource constraints and opportunity costs from the payers' point of view.

Evidence based medicine brings us to the question of power over the clinical encounter: will it be the doctor, the patient, or the payer who decides what kinds of treatment are delivered and on what basis? It is likely that this tension is contributing to the apparently growing frustration of doctors worldwide. ${ }^{33}$ Restating traditional medical ethics will not solve the problems because the economic crisis of medicine is here to stay, but requiring doctors to combine both traditional and modern duties is not a simple solution either. It is possible that the profession will split into two: doctors who can afford to follow traditional ethics with wealthy patients, and doctors trying to deliver the best possible care to poorer patients within the financial constraints imposed from above. This gap is likely to widen along with the scientific development and commodification of health care. ${ }^{34}$ Clinical practice guidelines have an internal logic that will make them more and more binding in the long run. It is also important to 
note the potential frustration of patients: paternalism in medicine has long been in decline and patient autonomy has been promoted to the extent that patients now tend to be called "clients" of health care. Evidence based medicine used for rationing will equally limit the autonomy of both the doctor and his/her clients. In conclusion, it would appear that using practical EBM for cost control may easily become the European equivalent of managed care.

In applying EBM to practice many important questions arise which cannot be resolved by further empirical research, but which require value based decisions. Being transparent and democratic on these points will allow us to use EBM better for both the good of individual patients and for rationing purposes. It will make the process of guideline creation fairer for all parties. Also, it will hopefully allow us to cope better with some of the fundamentally irresolvable conflicts that exist between the individual and the public health views, and between traditional medical ethics and the modern world. Using EBM as a powerfully attractive tool for disguising political programmes as science will only frustrate both doctors and patients.

\section{ACKNOWLEDGEMENTS}

We thank P Louhiala, S Rautava, and the two anonymous referees of Journal of Medical Ethics for their valuable comments on an earlier draft of this manuscript, and the Ahokas Foundation for economic support.

\section{Authors' affiliations}

S I Saarni, National Public Health Institute, Helsinki, Finland

H A Gylling, Department of Moral and Social Philosophy, University of Helsinki, Helsinki, Finland

SS has received a grant from the Ahokas Foundation, Finland.

\section{REFERENCES}

1 Grol R. Improving the quality of medical care. Building bridges among professional pride, payer profit and patient satisfaction. JAMA 2001;284:2578-85.

2 Tonelli MR. The philosophical limits of evidence based medicine. Acad Med 1998;73:1234-40.

3 Sackett DL, Rosenberg WMC, Gray JAM, et al. Evidence based medicine: what it is and what it isn't. BMJ 1996:312:71.

4 Tanenbaum SJ. Evidence and expertise: the challenge of the outcomes movement to medical professionalism. Acad Med 1999;74:757-63.

5 Clinicians for the Restoration of Autonomous Practice (CRAP) Writing Group. EBM: unmasking the ugly truth. BMJ 2002;325:1496-8.

6 Woolf SH, Grol R, Hutchinson A, et al. Potential benefits, limitations, and harms of clinical guidelines. BMJ 1999;318:527-30.
7 Veatch RM. Doctor does not know best: why in the new century physicians must stop trying to benefit patients. J Med Philos 2000;25:701-21.

8 Holm S. The second phase of priority setting. Goodbye to the simple solutions: the second phase of priority setting in health care. BMJ 1998;317:1000-2.

9 Cochrane AL. Effectiveness and efficiency. Random reflections on health services. Cambridge: Cambridge University Press, 1972.

10 Upshur REG. The ethics of alpha: reflections on statistics, evidence and values in medicine. Theor Med Bioeth 2001;22:565-76.

11 Harris J. Justice and equal opportunities in health care. Bioethics 1999;13:392-404.

12 Dowie J. Analysing health outcomes. J Med Ethics 2001;27:245-50.

13 Foy R, So J, Rous E, et al. Perspectives of commissioners and cancer specialists in prioritising new cancer drugs: impact of the evidence threshold. BMJ 1999;318:456-9.

14 Ham C. Tragic choices in health care: lessons from the child B case. BMJ 1999;319:1258-61.

15 Powers M. Managed care: how economic incentive reforms went wrong Kennedy Inst Ethics J 1997:7:353-60.

16 Sullivan WM. What is left of professionalism after managed care? Hastings Cent Rep 1999;29:7-13.

17 Medical Professionalism Project. Medical professionalism in the new millennium: a physicians' charter. Lancet 2002;359:520-2.

18 Sabin JE. The second phase of priority setting. Fairness as a problem of love and the heart: a clinician's perspective on priority setting. BMJ 1998;317:1002-4

19 Brennan TA. Luxury primary care-market innovation or threat to access? N Engl J Med 2002;346:1165-8.

20 Erdmann Y, Wilson R. Managed care: a view from Europe. Annu Rev Public Health $2001 ; 22: 273-91$.

21 Norheim OF. Healthcare rationing-are additional criteria needed for assessing evidence based clinical practice guidelines? BMJ 1999:319:1426-9.

22 Asch DA, Ubel PA. Rationing by any other name. N Engl J Med 1997;336: 1688-71.

23 Ham C, Coulter A. Explicit and implicit rationing: taking responsibility and avoiding blame for health care choices. J Health Serv Res Policy $2001 ; 6: 163-9$.

24 Daniels N, Sabin JE. Limits to healthcare: fair procedures, democratic deliberation, and the legitimacy problem for insurers. Philos Public Aff 1997;26:303-50.

25 Harbour R, Miller J. A new system for grading recommendations in evidence based guidelines. BMJ 2001;323:334-6.

26 The AGREE Collaboration. Appraisal of guidelines for research \& evaluation (AGREE) instrument. www.agreecollaboration.org 2001 (accessed 13 Feb 2004).

27 Shekelle $\mathbf{P}$, Woolf $S$, Eccles $M$, et al. Clinical guidelines: creating guidelines. BMJ 1999;318:593-6.

28 Black N. Evidence based policy-proceed with care. BMJ 2001;323:275-9.

29 Dent THS, Sadler M. From guidance to practice: why NICE is not enough. BMJ 2002;324:842-5.

30 Cookson R, McDaid D, Maynard A. Wrong SIGN, NICE mess: is national guidance distorting allocation of resources? BMJ 2001;323:743-5.

31 Hurwitz B. Clinical guidelines: legal and political considerations of clinical practice guidelines. BMJ 1999;318:661-4.

32 Lyles A. Direct marketing of pharmaceuticals to consumers. Annu Rev Public Health 2002;23:73-91.

33 Edwards N, Kornacki MJ, Silversin J. Unhappy doctors: what are the causes and what can be done? BMJ 2002;324:835-8.

34 Pellegrino ED. The commodification of medical and health care: the moral consequences of a paradigm shift from a professional to a market ethic. J Med Philos 1999;24:243-66. 